\title{
A Chronic Progressive Case of Enteroviral Limbic Encephalitis Associated with Autoantibody to Glutamate Receptor $\varepsilon 2$
}

\author{
Hideto Nakajima ${ }^{a}$ Mitsuaki Hosoya ${ }^{b}$ Yukitoshi Takahashi ${ }^{c} \quad$ Kuniko Matsuyama ${ }^{a}$ \\ Muneyoshi Tagami $^{a}$ Simon Ishida ${ }^{a}$ Daisuke Furutama ${ }^{a}$ Masakazu Sugino ${ }^{a}$ \\ Fumiharu Kimura $^{a}$ Kei-ichi Shinoda ${ }^{d}$ Toshiaki Hanafusa ${ }^{a}$ \\ ${ }^{a}$ Department of Internal Medicine I, Osaka Medical College, Takatsuki, ${ }^{b}$ Department of Pediatrics, \\ School of Medicine, Fukushima Medical University, Fukushima, ' Department of Pediatrics, \\ National Epilepsy Center, Shizuoka Institute of Epilepsy and Neurological Disorders, Shizuoka, and \\ ${ }^{\mathrm{d} D e p a r t m e n t}$ of Internal Medicine, Hanwa Sumiyosi Hospital, Osaka, Japan
}

Dear Sir,

Enteroviruses - coxsackieviruses and echoviruses - are frequent causes of aseptic meningitis, particularly in children. These viruses occasionally cause mild diffuse encephalitis but are rarely life threatening [1]. Herein, we describe a patient with chronic progressive limbic encephalitis caused by enterovirus, who was positive for autoantibodies against glutamate receptor (GluR) IgG- $\varepsilon 2$ in the cerebrospinal fluid (CSF), and in whom lesions were found in the bilateral hippocampus, medial temporal lobe and hypothalamus.

A 22-year-old right-handed male was referred to us in August 1995 because of fever, a 3-week history of sudden onset memory loss, repetitive questioning and subacute progressive mental confusion. He had no pyramidal or extrapyramidal disturbances or cerebellar ataxia. Fluid-attenuated inversion-recovery (FLAIR) MRI of the brain showed hyperintense lesions in the bilateral hippocampal structures (fig. 1A). No enhancement was seen after intravenous gadolinium. Routine laboratory tests, including complete blood count and blood chemistry, were normal. Serum antinuclear, anti-DNA, anti-Sm, anti-SSA/ SSB, anti-RNP, anti-Scl-70 and anti-Hu antibodies were negative. Analysis of CSF showed 5 cells $/ \mathrm{mm}^{3}$ (mononuclear), protein concentration $54 \mathrm{mg} / \mathrm{dl}$ and glucose $63 \mathrm{mg} / \mathrm{dl}$. PCR for herpes simplex virus (HSV) DNA was negative. He was treated with acyclovir (30 mg/kg/day for 14 days) and betamethasone (16 mg/day for 7 days) based on the diagnosis of limbic encephalitis. His symptoms and MRI abnormalities gradually disappeared by the second hospital month, and he was discharged without any sequelae.

In November 1995, the patient again experienced a week of fever, headache and disorientation. At second admission, the patient was disoriented with memory impairment, including anterograde and retrograde amnesia. His Mini-Mental State Examination score was 17/30. FLAIR MRI again revealed hyperintense lesions in the bilateral hippocampal structures (fig. 1B). CSF examination showed 6 cells $/ \mathrm{mm}^{3}$ (mononuclear), protein concentration 42 $\mathrm{mg} / \mathrm{dl}$ and glucose $56 \mathrm{mg} / \mathrm{dl}$. He improved again by treatment with acyclovir and betamethasone.

In January 1998, this patient first experienced a generalized seizure. In October 1998, the seizures increased in frequency, and he was admitted to our hospital. He had an amnestic syndrome and his attention was easily distracted by irrelevant environmental incidents. He also exhibited sleep apnea syndrome, and MRI showed expansion and swelling of a left temporal lobe lesion (fig. 1C). He was intubated and treated with acyclovir, betamethasone and an anticonvulsant. In February 1999, although the left temporal lobe lesion had reduced, an expanded lesion was observed in the right temporal lobe with gadolinium enhancement in the hypothalamus (fig. $1 \mathrm{D}, \mathrm{E})$. Based on the suspected diagnosis of secondary autoimmune encephalitis, mizoribine (a purine, antimetabolic, immunosuppressive agent) was added. After that, no relapse of the symptoms of encephalitis or change in MRI findings was observed. In March 2001, MRI demonstrated severe atrophy of the bilateral hippocampal gyrus and uncus (fig. 1F). He was alert and followed simple commands. However, he had severe impairment of memory retention, and he could not be extubated because of sleep apnea syndrome.

Through the course of illness, the patient was examined extensively for the causes of chronic progressive limbic en-

\section{KARGER}

Fax +4161306 1234 E-Mail karger@karger.ch www.karger.com
(C) 2007 S. Karger AG, Basel 0014-3022/07/0574-0238\$23.50/0

Accessible online at: www.karger.com/ene
Hideto Nakajima, MD

Department of Internal Medicine I, Division of Neurology, Osaka Medical College 2-7 Daigakumachi, Takatsuki

Osaka 569-8686 (Japan)

Tel. +81 72683 1221, Fax +81 72683 1801, E-Mail in1045@poh.osaka-med.ac.jp 


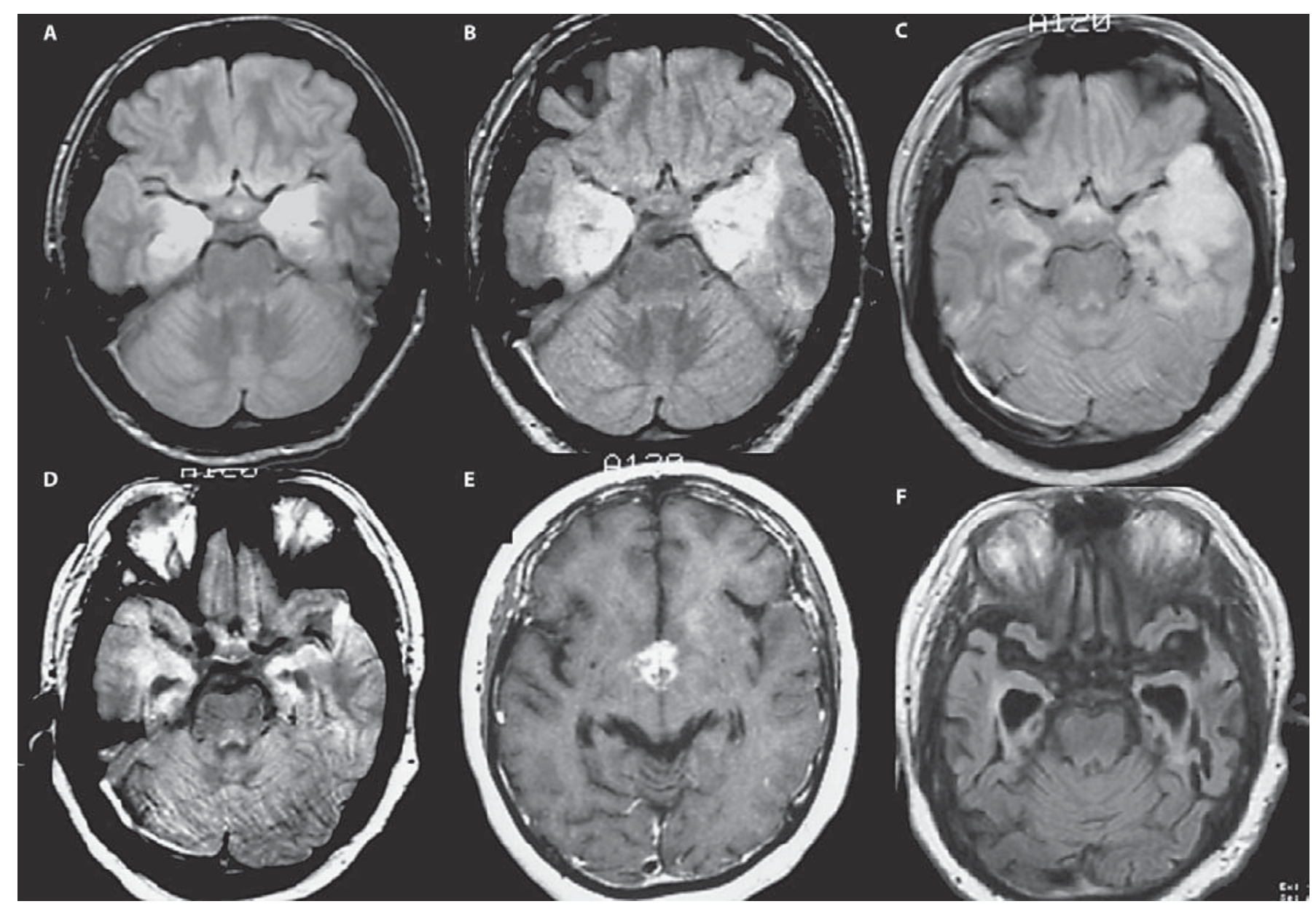

Fig. 1. Serial axial FLAIR MRI (A-D, F) and gadolinium-enhanced $T_{1}$-weighted image (E). Hyperintense lesions were seen in the bilateral hippocampus in August 1995 (A) and November 1995 (B). Expansion of the hyperintensity was seen in the left temporal lobe in January 1998 (C). In February 1999, hyperintensity was detected in the right temporal lobe and gadolinium enhancement in the hypothalamus (D, E). Severe atrophy of the bilateral hippocampus was observed in March 2001 (F).

cephalitis. He did not have any immunosuppressive disorders, such as HIV or agammaglobulinemia, and exhibited no abnormalities in either humoral or cell immunity. PCR studies of CSF were negative for HSV-1, HSV-2, cytomegaly, varicellazoster and human herpes virus 6. Measles, rubella and mumps were also serologically excluded. However, RT-PCR [2] revealed enterovirus RNA in 2 CSF samples obtained in August and November 1995. Although enterovirus RNA was not detected after 1998, 3 CSF samples exhibited positivity for autoantibodies against GluR IgG- $\varepsilon 2$ from 1998 to 1999 [3].

The nonpolio serotypes most often associated with central nervous system
(CNS) infection include echoviruses 7, 9, 11 and 30; coxsackievirus B5 and enterovirus 71 [1]. Although infection with enterovirus 71 has a high mortality rate, most patients with enteroviral CNS infection exhibit a mild, generalized disease that resolves without sequelae. Severe and chronic cases of focal encephalitis have been reported [4-6], even in immunocompromised hosts [7, 8]. Hokezu et al. [9] reported an immunocompetent patient with recurrent limbic encephalitis in which RT-PCR was able to detect echovirus 7 RNA in CSF samples. The present case was remarkable because chronic progressive limbic encephalitis and severe sequelae occurred in this patient without immunodeficiency and autoantibodies against GluR IgG- $\varepsilon 2$ being detected in the CSF of this patient. Limbic encephalitis is caused by HSV, nonherpetic viruses, paraneoplastic condition or autoimmune disorders. Recently, the presence of autoantibodies against GluR- $\varepsilon 2$ in the CSF has been suggested to be involved in the parainfectious autoimmune pathogenesis of limbic encephalitis. Autoantibodies against GluR- 22 may be associated with some symptoms of limibic encephalitis and sequelae (epilepsy and mental deterioration) of widespread encephalitis in childhood [10]. Moreover, they were speculated to be associated with atrophy of the hippocampus and impairment of memory. In 
this case, we presumed that autoantibodies against GluR IgG- $\varepsilon 2$ might have influenced the chronic progressive course. Enteroviral infection should be included in the differential diagnosis of limbic encephalitis. Furthermore, viral infections, such as enterovirus, can trigger the production of autoantibodies against GluR IgG- $\varepsilon 2$ in patients with limbic encephalitis.

\section{Acknowledgments}

This study was supported by Grantsin-Aid for Scientific Research (2006-7; 18590959) and Health and Labour Sciences Research Grants for Research on Psychiatry and Neurological Diseases and Mental Health (H17-017).

\section{References}

1 Rotbart HA: Enteroviral infections of the central nervous system. Clin Infect Dis 1995; 20:971-981.

2 Hosoya M, Honzumi K, Sato M, Katayose M, Kato K, Suzuki H: Application of PCR for various neurotropic viruses on the diagnosis of viral meningitis. J Clin Virol 1998;11:117124.

3 Takahashi Y, Mori H, Mishina M, Watanabe M, Fujiwara T, Shimomura J, Aiba H, Miyajima T, Saito Y, Nezu A, Nishida H, Imai K, Sakaguchi N, Kondo N: Autoantibodies to NMDA receptor in patients with chronic forms of epilepsia partialis continua. Neurology 2003;61:891-896.

4 Modlin JF, Dagan R, Berlin LE, Virshup DM, Yolken RH, Menegus M: Focal encephalitis with enterovirus infections. Pediatrics 1991; 88:841-845.
5 Zifroni A, Fried M, Shalmon B, Hoffman M, Yust I: Severe encephalitis with rapid recovery. Lancet 1997;349:1328.

6 Liow K, Spanaki MV, Boyer RS, Greenlee JE, Bale JF Jr: Bilateral hippocampal encephalitis caused by enteroviral infection. Pediatr Neurol 1999;21:836-838.

7 Cree BC, Bernardini GL, Hays AP, Lowe G: A fatal case of coxsackievirus B4 meningoencephalitis. Arch Neurol 2003;60:107-112.

8 McKinney RE Jr, Katz SL, Wilfert CM: Chronic enteroviral meningoencephalitis in agammaglobulinemic patients. Rev Infect Dis 1987;9:334-356.

9 Hokezu Y, Hashiguchi Y, Satake M, Hosoya M: A case report of recurrent limbic encephalitis caused by an enterovirus infection (in Japanese with English abstract). Rinsho Shinkeigaku 2004;44:165-170.

10 Takahashi Y: Infections as causative factors of epilepsy. Future Neurol 2006;1:291-302. 